University of Wollongong

Research Online

Australian Institute for Innovative Materials -

Papers

Australian Institute for Innovative Materials

2013

Microstructure and metal-dielectric transition behaviour in a percolative Al203-Fe composite via selective reduction

Zidong Zhang

Shandong University, University of Wollongong, zz842@uowmail.edu.au

Runhua Fan

Shandong University

Zhicheng Shi

Shandong University

Kelan Yan

Shandong University

Zhijia Zhang

University of Wollongong, zz755@uowmail.edu.au

See next page for additional authors

Follow this and additional works at: https://ro.uow.edu.au/aiimpapers

Part of the Engineering Commons, and the Physical Sciences and Mathematics Commons

Research Online is the open access institutional repository for the University of Wollongong. For further information contact the UOW Library: research-pubs@uow.edu.au 


\title{
Microstructure and metal-dielectric transition behaviour in a percolative Al203-Fe composite via selective reduction
}

\author{
Abstract \\ The electromagnetic (EM) medium plays a key role in many areas, such as communications, stealth \\ technology, etc. Different EM properties are required for different applications. In this paper, we have \\ obtained tunable EM properties in an Al2O3-Fe composite via selective reduction. By adjusting the \\ content of one functional component, the composite shows totally different EM properties, in accordance \\ with the predictions of effective medium theory. Hybrid EM behaviour is obtained near the percolation \\ threshold, which has a close relationship with its microstructure.

\section{Keywords} \\ percolative, metal, behaviour, microstructure, transition, reduction, dielectric, selective, via, composite, fe, \\ al2o3 \\ Disciplines \\ Engineering | Physical Sciences and Mathematics

\section{Publication Details} \\ Zhang, Z., Fan, R., Shi, Z., Yan, K., Zhang, Z., Wang, X. \& Dou, S. (2013). Microstructure and metal-dielectric \\ transition behaviour in a percolative Al2O3-Fe composite via selective reduction. RSC Advances, 3 (48), \\ 26110-26115.
}

\section{Authors}

Zidong Zhang, Runhua Fan, Zhicheng Shi, Kelan Yan, Zhijia Zhang, Xiaolin Wang, and S X. Dou 
Cite this: RSC Adv., 2013, 3, 26110

Received 20th September 2013

Accepted 22nd October 2013

DOI: $10.1039 / c 3 r a 45253 k$

www.rsc.org/advances

\section{Microstructure and metal-dielectric transition behaviour in a percolative $\mathrm{Al}_{2} \mathrm{O}_{3}-\mathrm{Fe}$ composite via selective reduction}

\author{
Zidong Zhang, ${ }^{\text {ab }}$ Runhua Fan, ${ }^{\text {*a }}$ Zhicheng Shi, ${ }^{a}$ Kelan Yan, ${ }^{a} Z^{2} i j i a ~ Z h a n g,{ }^{b}$ \\ Xiaolin Wang ${ }^{* b}$ and Shixue Dou ${ }^{b}$
}

The electromagnetic (EM) medium plays a key role in many areas, such as communications, stealth technology, etc. Different EM properties are required for different applications. In this paper, we have obtained tunable EM properties in an $\mathrm{Al}_{2} \mathrm{O}_{3}-\mathrm{Fe}$ composite via selective reduction. By adjusting the content of one functional component, the composite shows totally different EM properties, in accordance with the predictions of effective medium theory. Hybrid EM behaviour is obtained near the percolation threshold, which has a close relationship with its microstructure.

\section{Introduction}

The electromagnetic (EM) medium is becoming increasingly important with the rapid development of technology. Different types can be widely used in many fields, such as wireless communications, stealth technology, etc. ${ }^{1-3}$ According to the different applications, different EM properties are required., ${ }^{4,5}$ For example, in order to optimize the microwave absorption properties of a stealth aircraft's coating, both impedance matching and high energy loss should be taken into consideration. On the contrary, if we want to get good EM wave transparency, which is required in a nose radome, the energy loss should be kept at a relatively low level to maximize the signal.

As is well known, the response of the EM medium to the electromagnetic waves is largely determined by the permittivity $(\varepsilon)$ and the permeability $(\mu)^{6,7}$ For an aircraft's nose radome, in order to maximize the EM waves entering the material, the real and imaginary parts of the complex permittivity and permeability should be comparable, leading to good impedance matching between the material and free space. ${ }^{8}$ Otherwise, EM waves will be reflected back at the surface due to the impedance mismatching, which is the basic principle of electromagnetic shielding. ${ }^{9}$

With the development of modern technology, the demand for EM media with different properties has increased dramatically. Moreover, in some special high-technology fields, such as aerospace, electronics, and the military, the EM medium will

\footnotetext{
${ }^{a}$ Key Laboratory for Liquid-Solid Structural Evolution and Processing of Materials (Ministry of Education), Shandong University, Jinan 250061, China. E-mail: fan@ sdu.edu.cn; Tel: $+86-531-883-93396$

${ }^{b}$ Institute for Superconducting and Electronic Materials, University of Wollongong, Wollongong, New South Wales 2500, Australia. E-mail: xiaolin@uow.edu.au; Tel: $+61-2-4421-5766$
}

usually be used in an extreme environment, such as a hightemperature, high-pressure, and corrosive environment. It is impossible for a single material to have different properties to meet the demands of today's industry. ${ }^{10,11}$

Therefore, the electromagnetic composite plays a key role in today's EM field due to its various structural and functional properties, which originate from the components, and their distributions and morphologies, as well as the structure and composition of the interface between the components. ${ }^{12,13}$

In this paper, we report the fabrication of a new kind of EM composite obtained via a selective reduction. We use the selective reduction to refine the grains of the metal particles and use $\mathrm{Al}_{2} \mathrm{O}_{3}$ to control the concentration of free electrons, leading to tunable EM properties in the $\mathrm{MHz}$ range. In order to investigate the electromagnetic response of our samples, we use the Bruggeman effective medium theory (EMT) for calculations. ${ }^{14}$

The EM properties of our samples can be well described by the percolation theory and the effective medium theory. Near the percolation threshold, a metal-dielectric hybrid behaviour is obtained in the new EM composite, which has a close relationship to its microstructure and distribution of elements. The fundamentals of tunable EM properties will be discussed based on the experimental results and EMT calculations in this paper.

\section{Experiment}

$\mathrm{Fe}_{2} \mathrm{O}_{3} / \mathrm{Al}_{2} \mathrm{O}_{3}$ mixtures, with different $\mathrm{Al}_{2} \mathrm{O}_{3}$ molar ratios, were wet-milled in ethanol for 10 hours and dried at $373 \mathrm{~K}$ for 3 hours. Then, the powders were pressed under $40 \mathrm{kN}$ for $2 \mathrm{~min}$ to prepare plate-shaped samples $\left(15 \mathrm{~mm}^{2}\right.$ with thickness of $4 \mathrm{~mm}$ ), followed by pressureless sintering in air at $1573 \mathrm{~K}$ for 1.5 hours. The as-sintered products were placed in a tube furnace and isothermally reduced in hydrogen for 3 hours at different temperatures to yield the metal-ceramic composites. 
After the reduction process, different types of Fe-rich structures will be formed in the $\mathrm{Al}_{2} \mathrm{O}_{3}$ matrix with the $\mathrm{Al}_{2} \mathrm{O}_{3}$ molar ratios changing. For high $\mathrm{Al}_{2} \mathrm{O}_{3}$ content, island-like Fe-rich structure will be formed in the composite. As the $\mathrm{Al}_{2} \mathrm{O}_{3}$ content decreases, the Fe-rich island-like structures will be connected together to form a fishnet structure.

The phase identification of samples was performed by X-ray diffraction (XRD) using $\mathrm{Cu} \mathrm{K} \alpha$ radiation $(\lambda=0.15405 \mathrm{~nm}$ ). Mössbauer (MS) spectra were collected at room temperature, using a ${ }^{57}$ Co source contained in an Rh matrix. Both the real part and the imaginary part of the permittivity were measured by using an impedance analyzer (Agilent, 4991A) from $10 \mathrm{MHz}$ to $1 \mathrm{GHz}$.

\section{Phase characterization}

According to Todd's research, the reduction reaction of $\mathrm{Fe}_{2} \mathrm{O}_{3}$ will be affected by the temperature. ${ }^{15}$ In this paper, the

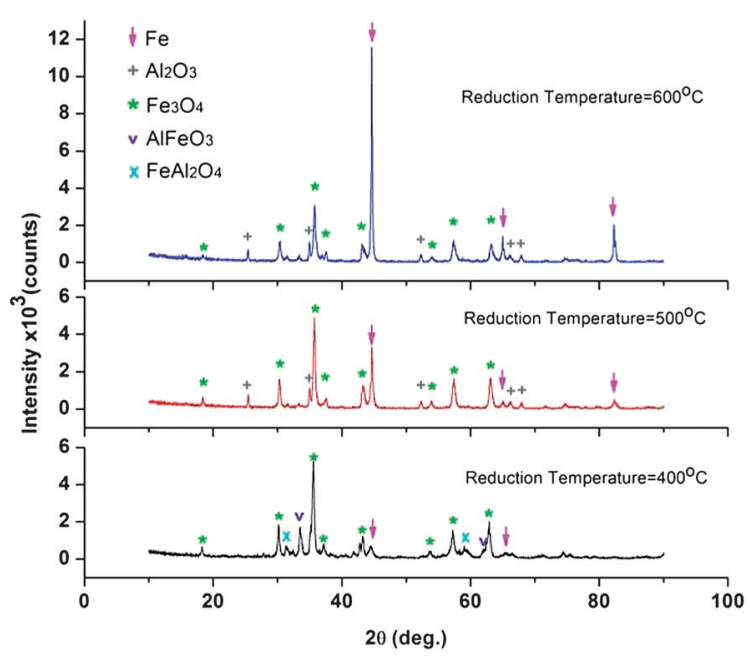

Fig. $1 \mathrm{X}$-ray diffraction patterns of the samples ( $50 \mathrm{~mol} \% \mathrm{Al}_{2} \mathrm{O}_{3}$ ) reduced at different temperatures in hydrogen for 3 hours.

Table 1 Parameters of specimens from Mössbauer spectra

\begin{tabular}{|c|c|c|c|c|c|}
\hline $\begin{array}{l}\text { Reduction } \\
\text { temperature }\left(T_{\mathrm{R}}\right)\end{array}$ & Subspectrum & $\begin{array}{l}\mathrm{IS} / \\
\mathrm{mm} \mathrm{s}^{-1}\end{array}$ & $\begin{array}{l}\mathrm{QS} / \\
\mathrm{mm} \mathrm{s}^{-1}\end{array}$ & $\begin{array}{l}\text { Magnetic } \\
\text { field } / \mathrm{T}\end{array}$ & $\mathrm{A} / \%$ \\
\hline \multirow[t]{5}{*}{$T_{\mathrm{R}}=600{ }^{\circ} \mathrm{C}$} & $\mathrm{Fe}^{2+}$-doublet & 0.96 & 1.78 & - & 5.5 \\
\hline & $\mathrm{Fe}^{3+}$-doublet & 0.23 & 0.77 & - & 6.7 \\
\hline & $\mathrm{Fe}_{3} \mathrm{O}_{4}$-sextet & 0.61 & -0.02 & 42.33 & 21.1 \\
\hline & $\mathrm{Fe}_{3} \mathrm{O}_{4}$-sextet & 0.33 & 0.02 & 46.42 & 21.9 \\
\hline & Fe-sextet & 0.00 & 0.00 & 33.07 & 44.8 \\
\hline \multirow[t]{5}{*}{$T_{\mathrm{R}}=500^{\circ} \mathrm{C}$} & $\mathrm{Fe}^{2+}$-doublet & 0.91 & 1.43 & - & 11.6 \\
\hline & $\mathrm{Fe}^{3+}$-doublet & 0.31 & 0.82 & - & 26.8 \\
\hline & $\mathrm{Fe}_{3} \mathrm{O}_{4}$-sextet & 0.31 & 0.03 & 47.78 & 14.5 \\
\hline & $\mathrm{Fe}_{3} \mathrm{O}_{4}$-sextet & 0.65 & -0.05 & 44.14 & 19.0 \\
\hline & Fe-sextet & 0.00 & 0.00 & 33.10 & 28.1 \\
\hline \multirow[t]{5}{*}{$T_{\mathrm{R}}=400^{\circ} \mathrm{C}$} & $\mathrm{Fe}^{2+}$-doublet & 0.85 & 1.27 & - & 10.4 \\
\hline & $\mathrm{Fe}^{3+}$-doublet & 0.26 & 0.79 & - & 26.6 \\
\hline & $\mathrm{Fe}_{3} \mathrm{O}_{4}$-sextet & 0.75 & 0.08 & 45.06 & 28.2 \\
\hline & $\mathrm{Fe}_{3} \mathrm{O}_{4}$-sextet & 0.23 & -0.09 & 46.77 & 26.5 \\
\hline & Fe-sextet & 0.00 & 0.00 & 33.00 & 8.3 \\
\hline
\end{tabular}

reduction processes were conducted at $600{ }^{\circ} \mathrm{C}, 500{ }^{\circ} \mathrm{C}$, and $400{ }^{\circ} \mathrm{C}$ by using pure hydrogen. The XRD patterns of the reduced samples (with $50 \mathrm{~mol} \% \mathrm{Al}_{2} \mathrm{O}_{3}$ ) are shown in Fig. 1. The peaks associated with $\mathrm{Fe}_{3} \mathrm{O}_{4}, \mathrm{Al}_{2} \mathrm{O}_{3}$, and $\mathrm{Fe}$ appeared after 3 hours reduction at $600{ }^{\circ} \mathrm{C}$. The phase composition did not change until we decreased the reduction temperature $\left(T_{\mathrm{R}}\right)$ to $400{ }^{\circ} \mathrm{C}$. New peaks corresponding to $\mathrm{AlFeO}_{3}$ and $\mathrm{FeAl}_{2} \mathrm{O}_{4}$ appeared, and no peaks of $\mathrm{Al}_{2} \mathrm{O}_{3}$ could be detected in the sample reduced at $400{ }^{\circ} \mathrm{C}$.
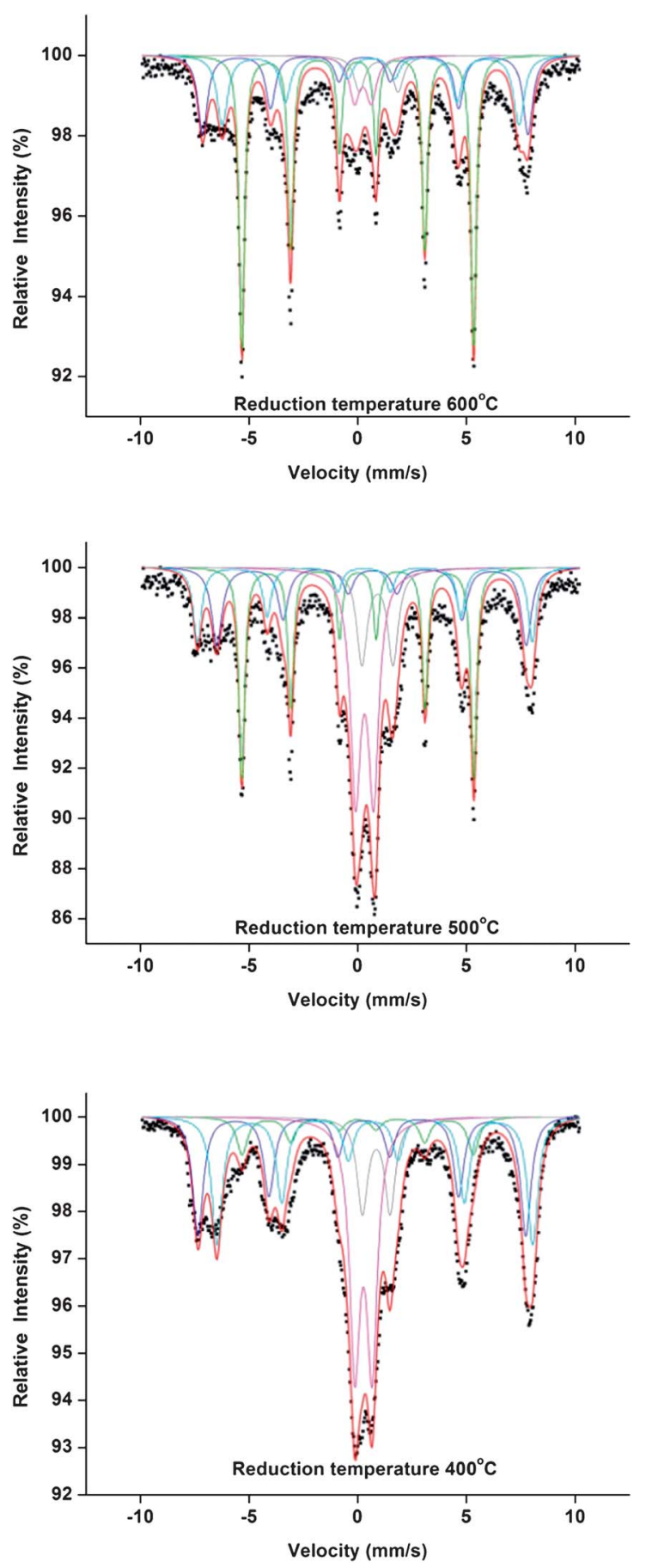

Fig. 2 Mössbauer spectroscopy patterns of the samples (50 mol\% $\mathrm{Al}_{2} \mathrm{O}_{3}$ ) reduced in hydrogen for 3 hours at different temperatures. 
Mössbauer (MS) spectra were collected at room temperature to obtain the accurate phase composition. The Mössbauer spectroscopy parameters are listed in Table 1, while Fig. 2 shows the Mössbauer spectroscopy (MS) pattern of each sample.

As shown in the MS results, all the samples displayed three sextets and two doublets. The sextet with an isomer shift (IS) of $0.00 \mathrm{~mm} \mathrm{~s}^{-1}$, quadrupole splitting (QS) of $0.00 \mathrm{~mm} \mathrm{~s}^{-1}$, and magnitude of the hyperfine field of $33 \mathrm{~T}$, can be identified as Fe. Meanwhile, the other two sextets can be associated with $\mathrm{Fe}_{3} \mathrm{O}_{4} \cdot{ }^{16}$ The characterization of the doublets becomes a little complex, however, because the ion transfer behaviour has a strong relationship with the reduction temperature. According to Laurent's research, $\mathrm{FeAl}_{2} \mathrm{O}_{4}$ phase will be formed during the reduction reaction of the $\mathrm{Fe}_{2} \mathrm{O}_{3} / \mathrm{Al}_{2} \mathrm{O}_{3}$ system in $\mathrm{H}_{2}$ at temperatures lower than $1000{ }^{\circ} \mathrm{C} .{ }^{17}$ In this case, the doublet with IS of $0.85-0.96 \mathrm{~mm} \mathrm{~s}^{-1}$ and QS of 1.27-1.78 $\mathrm{mm} \mathrm{s}^{-1}$ represents $\mathrm{Fe}^{2+}$ in the $\mathrm{FeAl}_{2} \mathrm{O}_{4}$ spinel structure. ${ }^{18,19}$ The other doublet is typical of $\mathrm{Fe}^{3+}$ ions substituting for $\mathrm{Al}^{3+}$ ions in an alumina lattice. ${ }^{20}$

\section{Electromagnetic results and discussion}

The frequency $(f)$ dispersions of the permittivity $(\varepsilon)$ are shown in Fig. 3 for the different reduction temperatures. Due to the limitations of the measurement device, however, an effective signal only can be collected from $10 \mathrm{MHz}$ to $1 \mathrm{GHz}$. As shown in Fig. 3, depending on the reduction temperature, the reduced samples show different EM properties. Based on the results, we can classify the reduced samples into two types.

The first type includes samples reduced at $500{ }^{\circ} \mathrm{C}$ and $600^{\circ} \mathrm{C}$. This type shows totally metal-like behaviour in the measurement range. Both the real and the imaginary parts of the permittivity can be well described by the Drude model. ${ }^{21}$

$$
\begin{aligned}
& \varepsilon(\omega)=1-\frac{\omega_{\mathrm{p}}{ }^{2}}{\omega^{2}+i \omega_{\tau} \omega}=\varepsilon^{\prime}+i \varepsilon^{\prime \prime} \\
& \varepsilon^{\prime}(\omega)=1-\frac{\omega_{\mathrm{p}}{ }^{2}}{\omega^{2}+\omega_{\tau}{ }^{2}}, \varepsilon^{\prime \prime}(\omega)=\frac{\omega_{\mathrm{p}}{ }^{2} \omega_{\tau}}{\omega^{3}+\omega_{\tau}{ }^{2} \omega}
\end{aligned}
$$
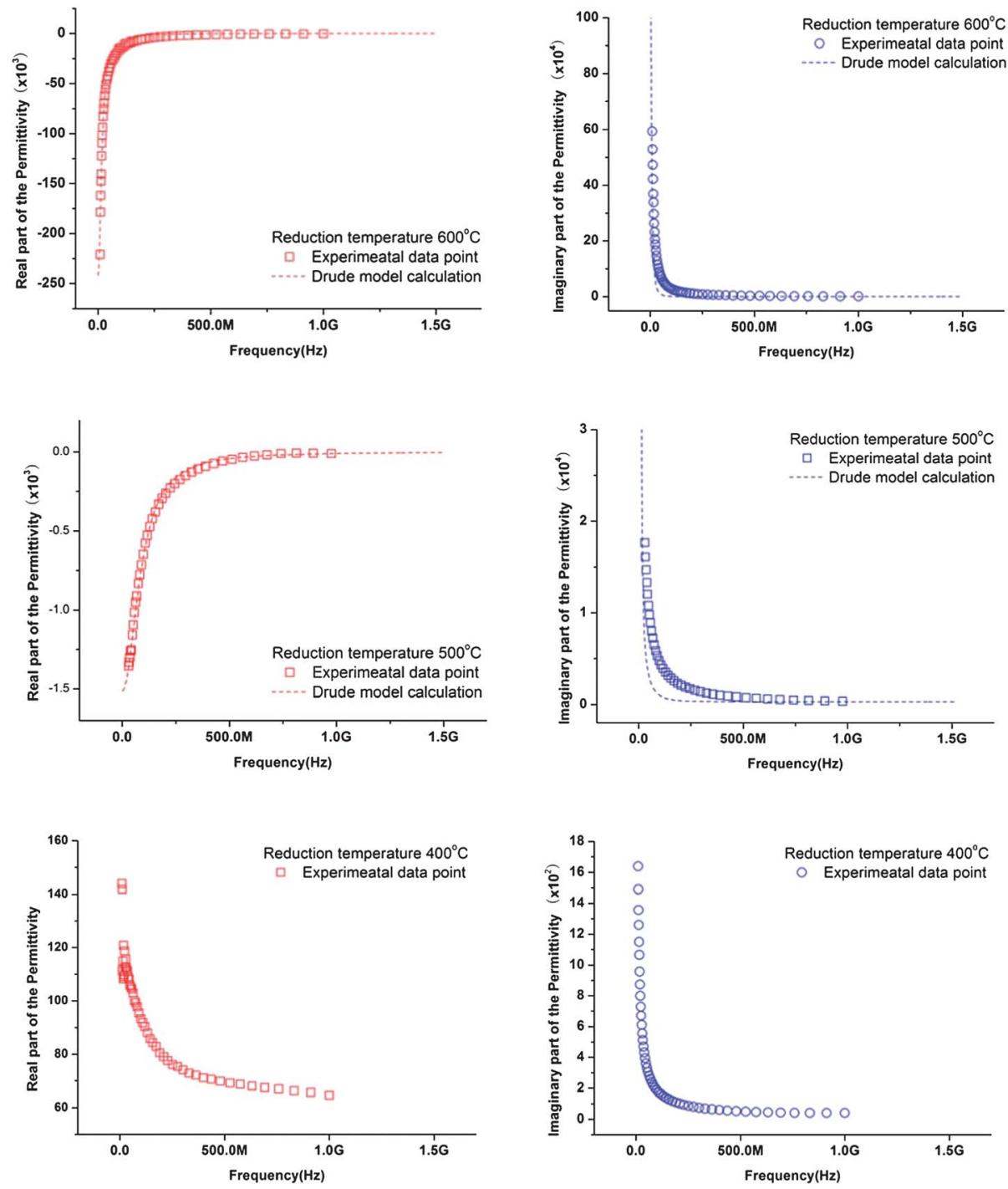

Fig. 3 Permittivity of the samples reduced at different temperatures. 


$$
\omega_{\mathrm{p}}=\sqrt{\frac{n e^{2}}{m_{\mathrm{eff}} \varepsilon_{0}}}
$$

where, $\omega_{\mathrm{p}}\left(2 \pi f_{\mathrm{p}}\right)$ is the plasma frequency, $\omega$ is the frequency of the electric field, $\omega_{\tau}$ is the damping parameter, $\varepsilon_{0}$ is the permittivity of vacuum $\left(8.85 \times 10^{-12} \mathrm{~F} \mathrm{~m}^{-1}\right), n$ is the bulk concentration of carriers, $m_{\mathrm{eff}}$ is the electron effective mass, and $e$ is the electron charge $\left(1.6 \times 10^{-19} \mathrm{C}\right)$.

The concentration of carriers in a metal usually remains on the order of $10^{23}$, which places the plasma frequency in the ultraviolet (UV) range (about $10^{15}-10^{17} \mathrm{~Hz}$ ). In our samples, the electron density is diluted by the non-conductive phase, which contributes to a reduced plasma frequency in the $\mathrm{GHz}$ range. The calculated results are also shown in Fig. 3 (dashed lines), which agree well with the experimental data. From the calculation, we can obtain the plasma frequencies of the samples reduced at $600{ }^{\circ} \mathrm{C}(8.6 \mathrm{GHz})$ and $500{ }^{\circ} \mathrm{C}(3.4 \mathrm{GHz})$. Based on Drude model calculation, we can predict the samples' EM properties. As shown in Fig. 3, the real part of the permittivity take a negative value in the measurement range, which suggest the EM wave will be reflected back in this range and the sample would be transparent to EM waves above the plasma frequency. ${ }^{13,22}$

We consider the sample reduced at $400{ }^{\circ} \mathrm{C}$ as typical of the second type, which can be called a metal-dielectric hybrid EM medium. As shown in Fig. 3, when we reduced the reduction temperature to $400{ }^{\circ} \mathrm{C}$, the real part of the permittivity became positive and could not be fitted by the Drude model, as it showed dielectric-like behaviour. Meanwhile, the imaginary part of the permittivity still retained a relatively high value, indicating metal-like behaviour.

According to the effective medium theory (EMT) predictions, the EM properties of metal-dielectric composites will cause them to act as dielectric media for low metal concentrations of less than the percolation threshold and act as dilute metals with an effective permittivity for concentrations beyond the percolation threshold. ${ }^{23}$ In our samples, as suggested by the MS results, the final phase composition can be controlled by changing the reduction temperature to obtain different contents of $\mathrm{Fe}$ (MS results) in each sample, leading to a different concentration of carriers $(n)$. For the samples reduced at $500{ }^{\circ} \mathrm{C}$ and $600{ }^{\circ} \mathrm{C}$ (the first type), as the content of conductive phase is beyond the percolation threshold, the medium can be considered as a dilute metal. ${ }^{24}$ Interestingly, both metal-like behaviour (large value of $\varepsilon^{\prime \prime}$ ) and dielectric-like behaviour (positive value of $\varepsilon^{\prime}$ ) are obtained in the sample reduced at $400^{\circ} \mathrm{C}$. This kind of EM property, which we have called the 'metal-dielectric hybrid EM property', is not included in the EMT predictions, however.

In order to reach a better understanding of this hybrid EM behaviour, we designed a new experiment to investigate the relationship between the content of conductive phase and the EM properties. Instead of changing the reduction temperature, we changed the content of $\mathrm{Al}_{2} \mathrm{O}_{3}$ in the $\mathrm{Fe}_{2} \mathrm{O}_{3} / \mathrm{Al}_{2} \mathrm{O}_{3}$ mixed powder. All the samples were reduced at $600{ }^{\circ} \mathrm{C}$ for 3 hours. The frequency $(f)$ dispersions of the permittivity $(\varepsilon)$ are shown in
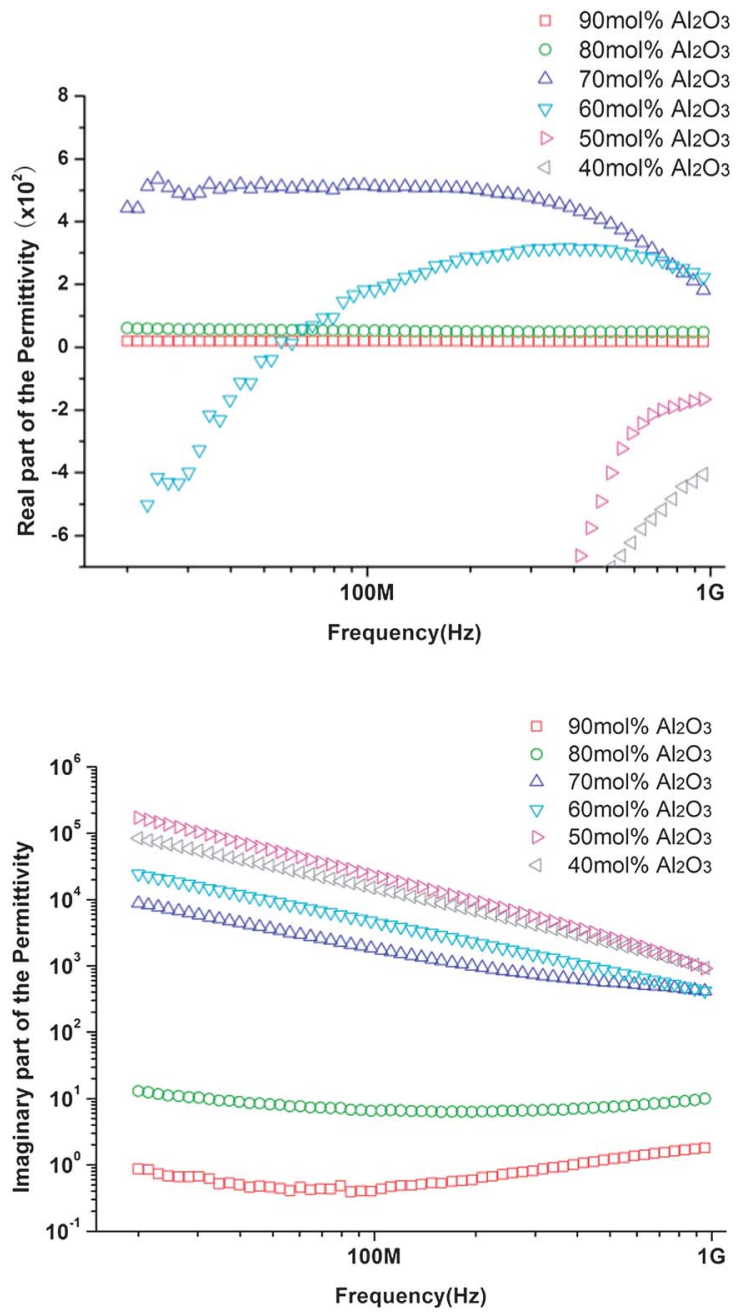

Fig. 4 Frequency dependence of the permittivity for different $\mathrm{Al}_{2} \mathrm{O}_{3}$ contents.

Fig. 4. As we can see in Fig. 4, both the real part and the imaginary part show a 'metal to dielectric' transition. As the content of $\mathrm{Al}_{2} \mathrm{O}_{3}$ is increased (from $40 \mathrm{~mol} \%$ to $90 \mathrm{~mol} \%$ ), the samples can be divided into two groups on the basis of the real part, a metal-like group ( $40 \mathrm{~mol} \%$ to $60 \mathrm{~mol} \% \mathrm{Al}_{2} \mathrm{O}_{3}$ ) and a dielectric-like group ( $70 \mathrm{~mol} \%$ to $90 \mathrm{~mol} \% \mathrm{Al}_{2} \mathrm{O}_{3}$ ). Similarly, these two groups are also reflected in the imaginary part (Table 2 and Fig. 4).

If we combine the real part with the imaginary part, depending on the $\mathrm{Al}_{2} \mathrm{O}_{3}$ content, the EM properties can be divided into three types, metal-like behaviour $(40 \mathrm{~mol} \%, 50 \mathrm{~mol}$ $\%$, and $60 \mathrm{~mol} \%$ ), metal-dielectric hybrid behaviour (70 mol\%) and dielectric-like behaviour ( $80 \mathrm{~mol} \%$ and $90 \mathrm{~mol} \%$ ). These

Table 2 Metal-like and dielectric-like group

\begin{tabular}{lll}
\hline & Metal-like group & Dielectric-like group \\
\hline$\varepsilon^{\prime}$ (Real part) & $40 \mathrm{~mol} \%, 50 \mathrm{~mol} \%$, & $70 \mathrm{~mol} \%, 80 \mathrm{~mol} \%$, \\
& $60 \mathrm{~mol} \%$ & $90 \mathrm{~mol} \%$ \\
$\varepsilon^{\prime \prime}$ (Imaginary part) & $40 \mathrm{~mol} \%, 50 \mathrm{~mol} \%$, & $80 \mathrm{~mol} \%, 90 \mathrm{~mol} \%$ \\
& $60 \mathrm{~mol} \%, 70 \mathrm{~mol} \%$ &
\end{tabular}


results clearly show a metal-dielectric transition following the EMT description and strongly suggest that the metal-dielectric hybrid EM property will be obtained near the percolation threshold.

The metal-like behaviour and dielectric-like behaviour can be easily understood according to the EMT, which has already been discussed in our earlier work. ${ }^{24}$ The most interesting part is the metal-dielectric hybrid EM property near the percolation threshold. Both EMT and the percolation theory suggest that the EM properties of a percolative composite have a close relationship with the microstructure. ${ }^{25}$

Backscattered electron images and element maps have been collected to find the relationship between the hybrid EM properties and the microstructure. Backscattered electron
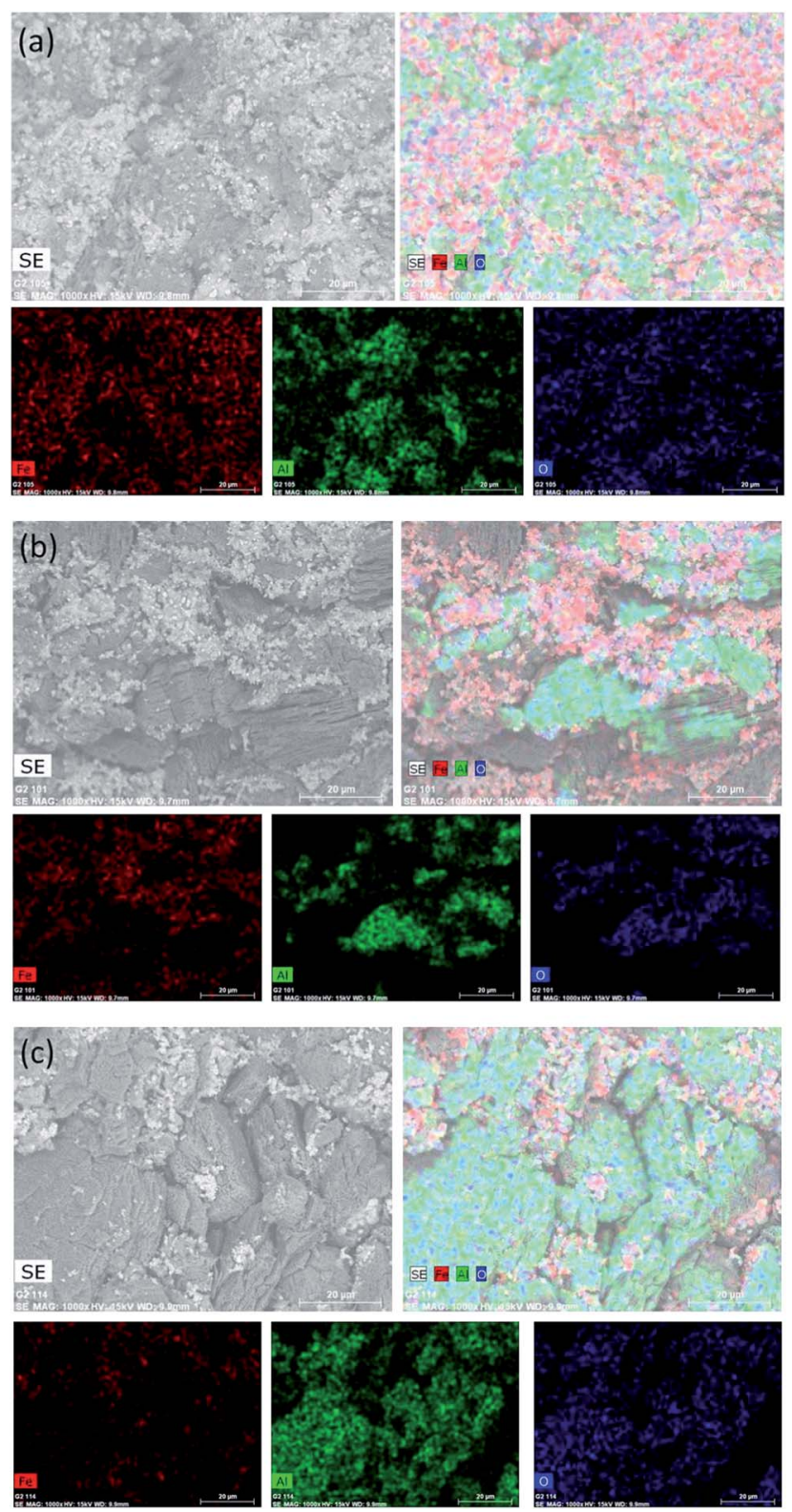

Fig. 5 Backscattered electron images and corresponding element distributions in (a) a metal-like composite $\left(40 \mathrm{~mol} \% \mathrm{Al}_{2} \mathrm{O}_{3}\right)$, (b) a metal-dielectric hybrid $\mathrm{EM}$ medium (70 mol\% $\mathrm{Al}_{2} \mathrm{O}_{3}$ ), (c) a dielectric-like composite (80 mol\% $\mathrm{Al}_{2} \mathrm{O}_{3}$ ). images and element distributions are shown in Fig. 5. In Fig. 5(a), the Fe-rich areas (red parts) can be considered as conductive paths that form a conductive network, which is spread throughout the whole matrix. Electrons can move freely in this network structure, in almost the same way as in a pure metal. That is the reason for the metal-like behaviour.

The whole conductive network will be cut into several local networks as the incorporation of $\mathrm{Al}_{2} \mathrm{O}_{3}$ is increased to $70 \mathrm{~mol} \%$. In this case, a new kind of microcapacitor will be formed in the composite. Each of the microcapacitors is formed by the neighboring local networks and the dielectric layers between them. The large capacitance contributed by each of the microcapacitors can then be correlated with a significant increase in the intensity of the local electric field, leading to a high dielectric constant (large value of the real part of the permittivity). At the same time, current still will be formed in the local network system, leading to a relatively high energy loss. As the mole ratio of $\mathrm{Al}_{2} \mathrm{O}_{3}$ reaches $80 \%$, the local network will be further divided into island-like structures surrounded by the insulating matrix and the microcapacitor structures will be destroyed, leading to a decrease of the real part of the permittivity. In this case, the free electrons are localized in the matrix and cannot move freely, resulting in a typical dielectric behaviour.

\section{Conclusions}

In this paper, percolative composites have been fabricated via a selective reduction reaction. The EM properties of the composites can be well described by effective medium theory and percolation theory. Near the percolation threshold, the composite shows an interesting metal-dielectric hybrid EM property, which has a close relationship with its microstructure and element distribution. A local conductive network leading to a large energy loss and a microcapacitor network significantly increases the dielectric constant of the composite.

The authors acknowledge the support of the National Natural Science Foundation of China (51172131), the Program for New Century Excellent Talents in University (NCET-10-518), the Provincial Science and Technology Development Project of Shandong (2007GG10003007, 2007BS04032), the Independent Innovation Foundation of Shandong University (IIFSDU2010JQ002, GIIFSDU-YYX10011), and the use of facilities within the University of Wollongong Electron Microscopy Centre. The authors thank Dr Tania Silver and Mrs Wenchao Yan for their critical reading of this manuscript, also Mr Xuchuan Wang for his help on the measurements.

\section{Notes and references}

1 J. R. Liu, M. Itoh, M. Terada, T. Horikawa and K. Machida, Appl. Phys. Lett., 2007, 91, 093101.

2 C. Sun and K. N. Sun, Solid State Commun., 2007, 141, 258.

3 X. G. Liu, D. Y. Geng and Z. D. Zhang, Appl. Phys. Lett., 2008, 92, 243110.

4 X. Zhang, O. Alloul, J. Zhu, Q. He, Z. Luo, H. A. Colorado, N. Haldolaarachchige, D. P. Young, T. D. Shen, S. Wei and Z. Guo, RSC Adv., 2013, 3(24), 9453. 
5 X. Zhang, Q. He, H. Gu, S. Wei and Z. Guo, J. Mater. Chem. C, 2013, 1(16), 2886.

6 H. Gu, J. Guo, X. Zhang, Q. He, Y. Huang, H. A. Colorado, N. Haldolaarachchige, H. Xin, D. P. Young, S. Wei and Z. Guo, J. Phys. Chem. C, 2013, $117(12), 6426$.

7 J. Zhu, X. Zhang, N. Haldolaarachchige, Q. Wang, Z. Luo, J. Ryu, D. P. Young, S. Wei and Z. Guo, J. Mater. Chem., 2012, 22(11), 4996.

8 Z. D. Zhang, Z. C. Shi, R. H. Fan, M. Gao, J. Y. Guo, X. G. Qi and K. N. Sun, Mater. Chem. Phys., 2011, 130, 615.

9 D. D. L. Chung, Carbon, 2012, 50, 3342.

10 D. D. L. Chung, Functional Composite Materials, in Advances in Condensed Matter and Materials Research, ed. F. Gerard, Nova Science Publ., Hauppauge, NY, 2003, p. 89.

11 D. D. L. Chung, Mater. Today, 2002, 5, 30.

12 H. Gu, Y. Huang, X. Zhang, Q. Wang, J. Zhu, L. Shao, N. Haldolaarachchige, D. P. Young, S. Wei and Z. Guo, Polymer, 2012, 53(3), 801.

13 X. Zhang, S. Wei, N. Haldolaarachchige, H. A. Colorado, Z. Luo, D. P. Young and Z. Guo, J. Phys. Chem. C, 2012, 116(29), 15731.

14 D. A. G. Bruggeman, Ann. Phys., 1935, 416, 636.

15 A. Mukhopadhyay and R. I. Todd, J. Eur. Ceram. Soc., 2010, 30, 1359.
16 A. Paesano and C. K. Matsuda, J. Magn. Magn. Mater., 2003, 264, 264.

17 A. Cordiera, A. Peigneya, E. De Graveb, E. Flahauta and C. Laurent, J. Eur. Ceram. Soc., 2006, 26, 3099.

18 J. H. Zhu, Z. P. Luo, S. J. Wu, N. Haldolaarachchige, D. P. Young, S. Y. Wei and Z. H. Guo, J. Mater. Chem., 2012, 22, 835.

19 C. Pecharroman and J. S. Moya, Adv. Mater., 2000, 12, 294.

20 V. G. de Resende, A. Cordier, E. De Grave, C. Laurent, S. G. Eeckhout, G. Giuli, A. Peigney, G. M. da Costa and R. E. Vandenberghe, J. Phys. Chem. C, 2008, 112, 16256.

21 Z. C. Shi, R. H. Fan, Z. D. Zhang, H. Y. Gong, J. Ouyang, Y. J. Bai, X. H. Zhang and L. W. Yin, Appl. Phys. Lett., 2011, 99, 032903.

22 C. Kittel, Introduction to solid state physics, Wiley, New York, 8th edn, 2004.

23 W. Cai, V. Shalaev, Optical Metamaterials: Fundamentals and Applications, Springer Science+Business Media, LLC, USA, 2010.

24 Z.-d. Zhang, R.-h. Fan, Z.-c. Shi, S.-b. Pan, K.-l. Yan, K.-n. Sun, J.-d. Zhang, X.-f. Liu, X. L. Wang and S. X. Dou, J. Mater. Chem. C, 2013, 1, 79.

25 C. W. Nan, Y. Shen and J. Ma, Annu. Rev. Mater. Res., 2010, 40, 131. 